\title{
Produção de crisântemos em diferentes substratos com e sem tratamento com promotor de crescimento(1)
}

\author{
LEANDRO OLIVEIRA DE ANDRADE(2); HANS RAJ GHEYI(3); FREDERICO ANTÔNIO LOUREIRO SOARES(4); \\ REGINALDO GOMES NOBRE ${ }^{(5)}$ e GEÓRGIA ROBERTA GOMES DE FIGUEIREDO( ${ }^{(6)}$
}

\begin{abstract}
RESUMO
No Brasil, a produção de flores tem crescido, ganhando importância econômica principalmente de crisântemos (Dendranthema grandiflora), que respondem por aproximadamente $80 \%$ do mercado interno de flores. Para a produção de mudas visando a plantas de boa qualidade, o substrato é fator básico, sobretudo no caso de culturas de propagação vegetativa. O objetivo deste trabalho foi avaliar quatro tipos de substratos, bem como o efeito do promotor de crescimento no enraizamento de estacas de crisântemo. O experimento foi conduzido em ambiente protegido, utilizando-se o delineamento em blocos casualizados, em esquema fatorial 4x2, com quatro repetições e duas mudas por repetição. Os fatores foram quatro substratos $\left(S_{1}-100 \%\right.$ de substrato comercial Topstrato Hortaliças ${ }^{\circledR}$; $\mathrm{S}_{2}-50 \%$, em medida de volume, de substrato comercial misturado com $50 \%$ de solo franco argiloso; $\mathrm{S}_{3}-55 \%$ de solo franco argiloso misturado com $15 \%$ de areia lavada, $15 \%$ de esterco bovino curtido e $15 \%$ de maravalha seca em base volume; $\mathrm{S}_{4}-25 \%$ de solo franco argiloso misturado com $25 \%$ de areia lavada, $25 \%$ de esterco bovino curtido e $25 \%$ de maravalha seca, em medida de volume), com e sem o pré-tratamento das estacas com promotor de crescimento vegetal Stimulate ${ }^{\circledR}$, visando a produzir mudas de crisântemo da cultivar Dobrado Sortido. Uma estaca contendo 5 folhas foi plantada em tubete plástico coberto com um telado negro de $50 \%$ de sombra e irrigado diariamente, 4 vezes ao dia. Ao final do período experimental, as mudas foram colhidas e as variáveis de crescimento, avaliadas. As análises de variância apresentaram efeito significativo $(\mathrm{p} \leq 0,01)$ dos substratos na altura de planta (AP) e no comprimento de raiz (CR). Os melhores resultados foram alcançados com o substrato $S_{2}$, que mostrou valores de AP 12,10; 31,45 e 30,24 e CR 2,75; 18,81 e $14,16 \%$ maiores quando comparado com os substratos $\mathrm{S}_{1}, \mathrm{~S}_{3}$ e $\mathrm{S}_{4}$, respectivamente. Por outro lado, as estacas sem pré-tratamento com Stimulate ${ }^{\circledR}$ mostraram melhor crescimento, apresentando efeitos significativos $(p \leq 0,05)$ no número de folhas e massas de matérias fresca e seca da parte aérea, raiz e total.
\end{abstract}

Palavras-chave: Dendranthema grandiflora, regulador de crescimento, enraizamento.

\section{ABSTRACT}

Production of chrysanthemum in different substrates with and without growth promoter pretreatment

The production of flowers has increased in Brazil and has gained more economic importance, especially the chrysanthemum (Dendranthema grandiflora), which responds for approximately $80 \%$ of the total internal flower market. The substrate is a basic input for the production of seedlings that results in plants of good quality, especially in vegetative propagated crops. A study was carried out under greenhouse conditions in randomized block design in $4 \times 2$ factorial scheme with the objective to test 4 substrates $\left(\mathrm{S}_{1}\right.$ - commercial substrate TOPSTRATO HORTALIÇAS ${ }^{\circledR} ; \mathrm{S}_{2}-50 \%$ of commercial substrate mixed with $50 \%$ of clay loam soil, $\mathrm{S}_{3}-55 \%$ of clay loam soil mixed with $15 \%$ of washed sand, $15 \%$ of cattle manure and $15 \%$ of wood shavings, and $\mathrm{S}_{4}-25 \%$ of clay loam soil mixed with 25 $\%$ of washed sand, $25 \%$ of cattle manure and $25 \%$ of wood shavings, all on volume basis) in combination with pretreatment of stakes with STIMULATE ${ }^{\circledR}$, growth promoter, in order to produce chrysanthemum cultivar Double Sorted seedlings. For analysis of the growth variables, chrysanthemum stakes with 5 leaves were planted individually in poly tubes. The poly tubes were covered with black screen of $50 \%$ shade and irrigated four times a day. At the end of experimental period the seedlings were harvested and growth variables were measured. Analysis of variance revealed significant effect $(\mathrm{p} \leq 0.05)$ of the substrates in plant height $(\mathrm{PH})$ and root length $(\mathrm{RL})$. Substrate $\mathrm{S}_{2}$ presented best results, the PH and RL under this treatment being 12.10, 31.45 and 30.24 \%, and 2.75, 18.81 and $14.16 \%$ greater than $\mathrm{S}_{1}, \mathrm{~S}_{3}$ and $\mathrm{S}_{4}$ substrates, respectively. On the other hand, the stakes without immersion in Stimulate ${ }^{\mathbb{}}$ showed better growth and significant effects $(\mathrm{p} \leq 0.05)$ were observed in case of number of leaves (NF), fresh and dry weights of shoot, root and total.

Keywords: Dendranthema grandiflora, growth regulator, seedling production.

\footnotetext{
(1) Parte da Dissertação de Mestrado do primeiro autor, financiada pela CAPES. . Recebido para publicação em 03/03/2009 e aceito em 21/07/2009

(2) Doutorando, Depto. Engenharia Agrícola, UFCG-CTRN, Campina Grande, PB. leandr.ufcg@hotmail.com

(3) Professor, Dr., Depto. de Engenharia Agrícola, UFCG-CTRN, Campina Grande, PB. hans@deag.ufcg.edu.br

(4) Bolsista Pós doutorado, CNPq, Depto. Engenharia Agrícola, UFCG-CTRN, Campina Grande, PB. fredalsoares@hotmail.com

${ }^{(5)}$ Bolsista Pós doutorado, CNPq, Depto. Engenharia Agrícola, UFCG-CTRN, Campina Grande, PB. rgomesnobre@yahoo.com.br

(6) Graduanda, Graduação em Agronomia, UFPB, Campus II, Areia, PB. Roberta_siegen@hotmail.com
} 


\section{INTRODUÇ̃̃̃O}

A floricultura no Brasil movimenta cerca de US\$ 800 milhões por ano e emprega de 15 a 20 pessoas por hectare com rendimento de $\mathrm{R} \$ 50$ a 100 mil (NEVES e AMARAL, 2007). Por outro lado, a profissionalização e o dinamismo comercial da floricultura brasileira são fenômenos relativamente recentes, mas que contabilizam números extremamente significativos em decorrência do enorme mercado interno de consumo. Há quatro anos, já se contava com mais de 2,6 mil produtores, cultivando, anualmente, uma área de cerca de 5,2 mil hectares (IBRAFLOR, 2004).

Segundo o BNB (2008), a floricultura é uma das cadeias produtivas vocacionadas do Nordeste do Brasil, que tem despertado interesse por parte de agentes produtivos, seja pelo seu potencial e sua produtividade, ou pela facilidade de produção e comercialização nacional e internacional. Por sua localização tropical, o Nordeste é uma das poucas regiões do mundo com possibilidades de produzir flores e plantas ornamentais com características exóticas, que obtêm preços diferenciados no mercado.

Como planta ornamental de grande destaque, está o cultivo do crisântemo, que, em 1996, já ocupava as primeiras posições no ranking do mercado interno, respondendo por aproximadamente $80 \%$ do total de flores comercializadas (FERNANDES et al., 2007).

A qualidade das plantas ornamentais cortadas depende, fundamentalmente, do tipo de substrato que se utiliza para cultivá-las e, em particular, das características físico-químicas desse substrato, já que o desenvolvimento e o funcionamento das raízes estão diretamente ligados às condições de aeração e ao conteúdo de água, apesar de ter influência direta sobre o fornecimento de suprimentos necessários para as espécies que nele se desenvolvem (ACOSTA-DURÁN et al., 2007). Todas estas interações se refletirão positiva ou negativamente na apresentação comercial das espécies cultivadas (ACOSTADURÁN et al., 2006). Portanto, o substrato é um insumo fundamental para o cultivo e a cadeia produtiva de flores, tornando-se um produto de grande destaque no mercado, sendo comercializado por inúmeras empresas que nele se especializaram (KÄMPF et al., 2006).

O substrato é o produto a ser usado em substituição ao solo na produção de plantas em recipientes e tem como função primordial promover suporte às plantas nele cultivadas, podendo ainda regular a disponibilidade de nutrientes e de água, além de permitir trocas gasosas das raízes (FONTENO, 1996; FERMINO et al., 2000; KÄMPF e FERMINO, 2000; RÖBER, 2000; GRUSZYNSKI, 2001). De forma geral, os substratos têm, em sua composição, matérias-primas de origem orgânica e inorgânica, responsáveis por manter as suas características fundamentais, que são: uniformidade, estabilidade química e da estrutura física, ausência de inibidores químicos de crescimento e de produtos tóxicos, teor de sais, capacidade de troca de cátions (CTC), densidade, custo, facilidade de manuseio, mistura e, por fim, facilidade de obtenção.

Contudo, a escolha do substrato deve considerar a grande diversidade de materiais e misturas utilizados, região produtora, tipo de produção, produtor (pequeno, médio ou grande), além da disponibilidade de matéria-prima e de substratos comerciais no mercado (SILVA, 2000) e custo.

$\mathrm{Na}$ floricultura, os reguladores ou estimulantes têm importância substancial, sendo muito utilizados para enraizamento, visando a elevar as produções, forçando as de entressafras, além de diminuir o porte das plantas, aumentar o número de flores por planta e alterar o tom das cores (YAMADA, 1992). SABBAGH (2008) verificou uma redução de corte de plantas de girassol ornamental ao utilizar reguladores de crescimento, assim como BRACKMANN et al. (2005), que comprovaram a teoria de que as giberelinas são compostos responsáveis pela aceleração da senescência, por acelerarem as funções de desenvolvimento e crescimento.

O objetivo deste trabalho foi avaliar o efeito do promotor de crescimento no enraizamento de estacas de crisântemo em diferentes substratos.

\section{MATERIAL E MÉTODOS}

Este experimento foi conduzido no período de maio a junho de 2008, em casa de vegetação do tipo capela, pertencente à Unidade Acadêmica de Engenharia Agrícola (UAEAg), da Universidade Federal de Campina Grande - UFCG, cujas coordenadas geográficas são: $7^{\circ} 15^{\prime} 18^{\prime \prime}$ de latitude sul, $35^{\circ} 52^{\prime} 28^{\prime \prime}$ de longitude oeste e altitude de $550 \mathrm{~m}$. O clima da região, conforme a classificação climática de Köppen, é do tipo As, que representa clima de Savana, tropical, com chuvas de inverno e verão seco. O local apresenta temperaturas médias máximas de $33{ }^{\circ} \mathrm{C}$ nos dias mais quentes de verão e $28^{\circ} \mathrm{C}$ em dias de inverno. As temperaturas médias mínimas ficam em torno de $23{ }^{\circ} \mathrm{C}$ nos dias mais quentes de verão, ou $15{ }^{\circ} \mathrm{C}$ nas noites mais frias do ano. A umidade relativa do ar está entre 75 e $82 \%$. Normalmente, o inverno começa em maio e termina em agosto.

Como método de propagação da variedade Dobrado Sortido de Crisântemo (Dendranthema grandiflora Tzvelev.), desenvolvida pela empresa Feltrin Sementes, utilizaram-se estacas com cortes de meristemas apicais com 5 folhas; as quais foram obtidas da parte superior da haste central de plantas com 30 dias de plantio, de produção própria (figura 1A).

As estacas foram submetidas a quatro tratamentos, os quais corresponderam a diferentes tipos de substrato $\left(\mathrm{S}_{1}-100 \%\right.$ de substrato comercial Topstrato Hortaliças ${ }^{\circledR} ; \mathrm{S}_{2}-50 \%$, em base de volume, de substrato comercial misturado com $50 \%$ de solo franco argiloso; $\mathrm{S}_{3}-55 \%$ de solo franco argiloso misturado com $15 \%$ de areia lavada, $15 \%$ de esterco bovino curtido e $15 \%$ de maravalha seca, em medida de volume; $\mathrm{S}_{4}-25 \%$ de solo franco argiloso misturado com $25 \%$ de areia lavada, $25 \%$ de esterco bovino curtido e $25 \%$ de maravalha seca) combinados com 2 níveis de indutor de crescimento, 0 e $0,02 \%$ de Stimulate $^{\circledR}$, que, por sua vez, é composto de $0,009 \%$ de cinetina, $0,005 \%$ de ácido giberélico e $0,005 \%$ de ácido indolbutírico e $99,981 \%$ de ingredientes inertes, conforme informa a STOLLER DO BRASIL (1998). As principais características dos substratos utilizados estão descritas na tabela 1.

O substrato comercial tinha na sua composição superfosfato simples, nitrato de potássio, turfa, vermiculita e casca de pinus, 
enquanto que a areia lavada foi adquirida em casa comercial de materiais de construção; a maravalha, que é um resíduo celulósico, proveio da indústria madeireira de móveis, e o esterco bovino curtido foi comprado em lojas de plantas ornamentais.

O material de solo empregado nas formulações dos substratos proveio de um Latossolo franco argiloso, coletado na camada superficial $(0-20 \mathrm{~cm})$ de uma área localizada no município de Campina Grande, o qual foi destorroado, homogeneizado, passado em peneira com malha igual a $5 \mathrm{~mm}$ e posto para secar ao ar; após secagem, o material foi caracterizado (tabela 2) no Laboratório de Irrigação e Salinidade da Universidade Federal de Campina Grande - LIS, utilizando métodos recomendados pela EMBRAPA (1997).

Após preenchimento com o respectivo substrato, os 64 tubetes de plástico ( $250 \mathrm{~mL}$ de capacidade), de cor preta, foram devidamente identificados e colocados em uma estante metálica apropriada, com altura de $37,0 \mathrm{~cm}$, possuindo 252 células e cobertura de malha preta $(50 \%)$, com a finalidade de oferecer as condições ideais (temperatura, umidade, luminosidade) durante todo o experimento (figura 1).

No caso do tratamento com o estimulante, as estacas foram imersas, durante 10 segundos, em solução de Stimulate ${ }^{\circledR}$, na concentração de $0,02 \%$ e, em seguida, plantadas.

As principais pragas detectadas durante o experimento foram a mosca-branca (Bemisia tabaci raça B) e a larvaminadora (Lyriomyza huidobrensis), que foram combatidas, logo após seu aparecimento, com pulverizações de Confidor $700 \mathrm{WG}$, na diluição recomendada $\left(360 \mathrm{~g} \mathrm{ha}^{-1}\right)$, e Abamectina, de nome comercial Vertimec $18 \mathrm{CE}$, na diluição recomendada (50 mL $100 \mathrm{~L}^{-1}$ de calda), intercalando com ciromazina, de nome comercial Trigard $750 \mathrm{WP}$, na concentração indicada (15 g $100 \mathrm{~L}^{-1}$ de calda). Esses produtos também serviram de controle preventivo para o tripes (Thrips palmi) e para o ácaro-rajado (Tetranychus urticae).

As mudas foram irrigadas diariamente, 4 vezes por dia, de modo que pudessem estar constantemente em solo com capacidade de campo ou próximo a este ponto. O molhamento do assoalho da casa de vegetação, durante o período do dia, foi necessário nos 15 primeiros dias, em virtude do período crítico para a planta e das altas temperaturas (entre 23 e $36^{\circ} \mathrm{C}$ ) do ambiente interno da casa de vegetação.

As variáveis avaliadas no final do experimento foram altura de plantas (AP) (a partir do nível do solo até o último nó da haste central), número de folhas (NF) (folhas com comprimento $>5 \mathrm{~cm}$ ), comprimento de raiz $(\mathrm{CR})$ (medição do comprimento da maior porção radicular desde o nível do solo até o final da maior raiz), massas de matéria fresca da parte aérea (FFPA), da raiz (FFR) e total (FFT), que correspondeu ao somatório das anteriores, massas de matéria seca da parte aérea (FSPA), da raiz (FSR) e total (FST).

O delineamento experimental adotado foi o de blocos casualizados em esquema fatorial 4 x 2, com 4 repetições e 2 mudas por repetição. Os efeitos da utilização dos diferentes substratos e da aplicação do estimulante químico sobre o crescimento de mudas de crisântemo foram avaliados mediante análise de variância (teste F), e suas médias foram comparadas pelo teste de Tukey a 0,01 de probabilidade, após transformações em $\sqrt{ } \mathrm{x}+1$, para todas as variáveis analisadas.

\section{RESULTADOS E DISCUSSÃO}

O efeito do fator tipo de substrato $(\mathrm{S})$ foi significativo no nível de 0,01 de probabilidade na altura de planta e no comprimento da raiz (tabela 3). Observou-se, no fator estimulante (E), que o efeito foi significativo apenas para número de folhas (NF), enquanto a interação $\mathrm{S} x \mathrm{E}$ não foi significativa para nenhuma das variáveis estudadas, indicando que o comportamento das plântulas em diferentes substratos não variou com a aplicação de estimulante químico.

A maior AP dos crisântemos foi obtida quando se utilizou substrato $\mathrm{S}_{2}$ (50\% comercial $+50 \%$ solo franco-argiloso), a qual diferiu significativamente de $S_{3}$ e $S_{4}$ e não diferiu de $S_{1}$; por outro lado, a menor AP foi obtida nas plantas cultivadas no substrato $\mathrm{S}_{3}$, apresentando decréscimo de $31,45 \%$ em relação ao substrato $\mathrm{S}_{2}$ (figura 2). TRANI et al. (2004), estudando a produção de mudas de alface em bandejas e substratos comerciais, observaram diferenças significativas quanto à altura média de plantas; já RAMOS et al. (2008) constataram, na produção de mudas de tomate, para variáveis avaliadas incluindo-se AP, que o substrato comercial Hortimix ${ }^{\circledR}$ proporcionou os melhores resultados, comparado com fibra de coco e resíduo de cultivo de cogumelo, respectivamente.

ZIETEMANN e ROBERTO (2007), estudando o efeito de diferentes substratos, casca de arroz carbonizado e vermiculita de granulação média, e épocas de coleta no enraizamento de estacas herbáceas de goiabeira cvs. Paluma e Século XXI, constataram diferença significativa no comprimento da raiz entre os substratos. SMARSI et al. (2008) também observaram diferença significativa no comprimento da raiz ao avaliarem concentrações de ácido indolbutírico e tipos de substrato na propagação vegetativa de lichia.

Provavelmente, o substrato $\mathrm{S}_{2}$, com $50 \%$ de substrato comercial misturado com $50 \%$ de solo franco-argiloso, o qual promoveu os melhores resultados, possui a vantagem de ter qualidades físicas (retenção de água e proporção de ar) apropriadas para produção de mudas em larga escala, além de ter baixo custo e facilidade de obtenção pelo produtor; bem como características fitossanitárias ideais decorrentes da proporção do substrato comercial nele contido. Além disso, é possível que $\mathrm{S}_{1}$ tenha retido excesso de água, comprometendo sua aeração, já que os substratos foram irrigados 4 vezes por dia, mantendo-o em sua capacidade de campo constantemente.

Nota-se que, apesar de não existirem diferenças significativas entre os substratos, o NF das plantas variou $11,5 \%$ entre o maior $\left(4,07\right.$ folhas planta $\left.^{-1}\right)$ e o menor $\left(3,65\right.$ folhas planta $\left.^{-1}\right)$; por outro lado, as estacas sem uso do estimulante $\left(\mathrm{E}_{1}\right)$ apresentaram um aumento de $8,47 \%$ (figura 3). Apesar de o mesmo comportamento, conforme já comentado, não se manifestar na análise das variáveis AP e CR, observa-se, em relação a AP, um decréscimo de $12,1 \%$ das mudas com utilização de estimulante $\left(E_{2}\right)$ em comparação com as sem utilização $\left(E_{1}\right)$ e em relação a $\mathrm{CR}$, um decréscimo de $\mathrm{E}_{1}$ para $\mathrm{E}_{2}$ de $7,8 \%$ (figura 3).

Para as variáveis massas de matérias fresca e seca da parte aérea, da raiz e total, não ocorreu efeito significativo dos substratos e da interação S x E (tabela 4). Comportamento contrário foi verificado em mudas de tomateiro, em que o substrato comercial foi o melhor em comparação com os 
alternativos (fibra de coco e resíduo do cultivo de cogumelos), porém, nesse mesmo experimento, para as variáveis massa de matéria seca de raiz e parte aérea, o composto com $100 \%$ de resíduo de cultivo de cogumelo (RCC) mostrou melhores médias; já para massa de matéria fresca de parte aérea, a melhor média obtida foi promovida pelo tratamento do substrato $20 \%$ de fibra de coco (FC) $+80 \%$ RCC, e, finalmente, o tratamento $40 \% \mathrm{FC}+60 \% \mathrm{RCC}$ mostrou melhor média em massa de matéria fresca de raiz (RAMOS et al., 2008). Por sua vez, os tratamentos com e sem estimulantes diferiram estatisticamente entre si (tabela 4); vários estudos, dentre outros, o de ZIETEMANN \& ROBERTO (2007), não obtiveram diferença significativa entre os diversos substratos utilizados na quantidade de matérias fresca e seca da raiz, em experimento desenvolvido com goiabeira.

Em todas as variáveis de matéria fresca, a melhor resposta foi verificada na ausência do estimulante $\left(\mathrm{E}_{1}\right)$ comparado com a sua presença. A vantagem de $E_{1}$ em relação a $E_{2}$, nos parâmetros de massas de matéria fresca da parte aérea, da raiz e total foi de 19,4, 10,4 e 20,83\%, respectivamente (figura 4).

Em relação à matéria seca, observa-se uma similaridade com a matéria fresca, porém em menor proporção, sendo que plantas submetidas a $E_{1}$ em relação a $E_{2}$ foram superiores em 2,7, 1,0 e 4,4\%, respectivamente, nos parâmetros massas de matéria seca da parte aérea, da raiz e total. Este resultado não era esperado, visto que o indutor de crescimento Stimulate ${ }^{\circledR}$ apresenta efeito sinergístico, em função da presença equilibrada dos reguladores de crescimento. Provavelmente, a baixa concentração e o curto tempo de imersão das estacas na solução de Stimulate ${ }^{\circledR}$ podem ter promovido esta resposta. $\mathrm{O}$ efeito do Stimulate ${ }^{\circledR}$ no alongamento celular foi verificado por VIEIRA \& CASTRO (2001), trabalhando com sementes de soja (Glycine $\max (\mathrm{L})$. Merril cv. IAC 8-2), sendo que concentração de $1,3 \mathrm{~mL}$ por $0,5 \mathrm{~kg}$ de sementes favoreceu o crescimento radicular vertical, com incremento de 9,9\%, em relação ao controle. Resultado semelhante foi observado em algodão cv. CNPAITA 90, em que a aplicação de Stimulate ${ }^{\circledR}$ a $8,5 \mathrm{~mL} 0,5 \mathrm{~kg}^{-1}$ de sementes promoveu um crescimento radicular vertical superior em $13,6 \%$ em relação ao controle (VIEIRA \& SANTOS, 2005). VIEIRA (2001), estudando a ação do Stimulate ${ }^{\circledR}$ em sementes de arroz e feijão, observou que o comprimento total do sistema radicular foi superior em $37,7 \%$ para a dose de $2,3 \mathrm{~mL}$ de Stimulate $^{\circledR}$ nas plantas de arroz e $19,8 \%$ para a dose de $5,0 \mathrm{~mL}$ de Stimulate ${ }^{\circledR} 0,5 \mathrm{~kg}^{-1}$ de sementes, no feijoeiro. REGHIN et al. (2000), em trabalho realizado com mandioquinha-salsa (Arracacia xanthorriza Bancroft), constataram efeito significativo do Stimulate ${ }^{\circledR}$ no número e comprimento de raízes de acordo com o aumento da dose até o limite de 7,0 $\mathrm{mL} \mathrm{L}^{-1}$, indicando ser o bioestimulante responsável pelo estímulo do crescimento e desenvolvimento radicular.

Ao contrário deste experimento, LEITE \& MARTINS (2007), ao avaliarem o efeito de estimulante de enraizamento, ácido indol butírico, em estacas semi-lenhosas do cacaueiro, com 30 dias, obtiveram maior quantidade de matéria seca da raiz ao utilizarem doses maiores de estimulantes.

\section{AGRADECIMENTOS}

À CAPES, pela concessão da bolsa de estudos.

\section{REFERÊNCIAS}

ACOSTA-DURÁN, C. M., LÓPEZ-MARTÍNEZ, V., ALIATEJACAL, I. Caracterización de materiales para sustrato de plantas em contenedor. Actas de Horticultura, Leuven, v.713, p.27-33, 2006.

ACOSTA-DURÁN, C. M., VARGAS-LÓPEZ, S., ACOSTAPENLOZA, D., NAVA-GÓMEZ, L. M., VILLEGASTORRES, O. G., ANDRADE-RODRÍGUEZ, M. Sustratos para la producción de crisantemo (Dendranthema grandiflora) em contenedor em México. Proceedings of Interamerican Society for Tropical Horticulture, Espírito Santo, v.51, p.298301, 2007.

BNB - BANCO DO NORDESTE DO BRASIL S. A. Cresce Nordeste - Floricultura. Net. Floricultura. Disponível em: http: //www.bnb.gov.br/content/aplicacao/Produtos_e_Servicos/ Cresce_Nordeste/gerados/cresce_nordeste_floricultura.asp?id $\mathrm{TR}=$ crescene. Acesso em: 21/01/2008.

BRACKMANN, A., BELLÉ, R. A., FREITAS, S. T., MELLO, A. M. Qualidade pós-colheita de crisântemos (Dedranthema grandiflora) mantidos em soluções de ácido giberélico. Ciência Rural, Santa Maria, v.35, n.6, 2005.

EMBRAPA. Centro Nacional e Pesquisas de Solos. Manual de métodos de análise do solo. Rio de Janeiro. Serviço de Levantamento e Conservação do Solo, 1997. 212p.

FERMINO, M. H., TRENTIN, A. L., KÄMPF, A. N. Caracterização física e química de materiais alternativos para composição de substratos para plantas: 1. Resíduos industriais e agrícolas. In: KÄMPF, A. N., FERMINO, M. H. (ed.). Substratos para plantas: a base da produção vegetal em recipientes. Porto Alegre: Genesis, 2000. p.241-248.

FERNANDES, E. P., SOUZA, E. R. B., LEANDRO, W. M., PIRES, L. L., VERA, R., SOUZA, R. F. Marcha de acúmulo de fitomassa em crisântemo (Dendranthema grandiflorum T., var. SALMON REAGAN). Pesquisa Agropecuária Tropical, Goiânia, v.37, n.3, p.137-141, 2007.

FONTENO, W. C. Growing media: Types and physical/ chemical properties. In: REED, D.W. (ed.) A grower's guide to water, media, and nutrition for greenhouse crops. Batavia: Ball, 1996. p.93-122.

GRUSZYNSKI, C. Produção comercial de crisântemos: vaso, corte e jardim. Uberaba. Editora Agropecuária, 2001. 166p. 
IBRAFLOR - INSTITUTO BRASILEIRO DE FLORICULTURA. Programa florabrasilis. Release Florabrasilis 2003/2004. Net. Disponível em: http:// www.ibraflor.com.br. Acesso em: 25/08/2004.

KÄMPF, A. N., FERMINO, M. A. Substratos para plantas: a base da produção vegetal em recipientes. Porto Alegre: Gênesis, 2000. 312p.

KÄMPF, A. N., TAKANE, R. J., SIQUEIRA, P. T. V. Floricultura: técnicas de preparo de substratos. Brasília: LK Editora e Comunicação, 2006. 132p.

LEITE, J. B. V., MARTINS, A. B. G. Efeito do ácido indolbutírico e época de coleta no enraizamento de estacas semi-lenhosas do cacaueiro. Revista Brasileira de Fruticultura, Jaboticabal, v.29, n.2, p.204-208, 2007.

NEVES, M. F., AMARAL, M. O. Flores - Oportunidades e desafios. Revista AgroAnalisys. Net. São Paulo. Set. 2007. Disponível em: http://www.agroanalysis.com.br/index.php? area $=$ conteudoemat_id $=327$ efrom $=$ mercadonegocios\#envie. Acesso em: 05/05/2008

RAMOS, S. J., GUILHERME, D. O., CALDEIRA JÚNIOR, C. F., SAMPAIO, R. A., COSTA, C. A., FERNANDES, L. A. Tomato seedling production in substrate containing coconut fiber and mushroom culture waste. Revista Brasileira de Ciências Agrárias, Recife, v.3, n.3, p.237-241, 2008.

REGHIN, M. Y., OTTO, R. F., SILVA, J. B. C. "Stimulate ${ }^{\circledR}$ Mo" e proteção com tecido "não tecido" no pré-enraizamento de mudas de mandioquinha-salsa. Horticultura Brasileira, Brasília, v.18, n.1, p.53-57, 2000.

RÖBER, R. Substratos hortícolas: possibilidades e limites de sua composição e uso; exemplos da pesquisa, da indústria e do consumo. In: KÄMPF, A. N., FERMINO, M. H. (ed.). Substratos para plantas: a base da produção vegetal em recipientes. Porto Alegre: Genesis, 2000. p.209-215.

SABBAGH, M. C. Redução de porte de girassol ornamental pela aplicação de reguladores vegetais. Curitiba: Departamento de Fitotecnia e Sanitarismo, Universidade Federal do Paraná, 2008. 93p. Dissertação (Mestrado em Agronomia).
SILVA, L. J. C. A demanda de substrato na floricultura. In: KÄMPF, A. N., FERMINO, M. H. (ed.). Substratos para plantas: a base da produção vegetal em recipientes. Porto Alegre: Genesis, 2000. p.163-165.

SMARSI, R. C., CHAGAS, E. A., REIS, L. L., OLIVEIRA, G. F., MENDONÇA, V., TROPALDI, L., PIO, R., SCARPARE

FILHO, J. A. Concentrações de ácido indolbutírico e tipos de substrato na propagação de lichia. Revista Brasileira de Fruticultura, Jaboticabal, v.30, n.1, p.7-11, 2008.

STOLLER DO BRASIL. Stimulate ${ }^{\circledR}$ Mo em hortaliças: Informativo técnico. Cosmópolis: Stoller do Brasil, Divisão Arbore, 1998.

TRANI, P. E., NOVO, M. C. S. S., CAVALLARO JÚNIOR, M. L., TELLES, L. M. G. Produção de mudas de alface em bandejas e substratos comerciais. Horticultura Brasileira, Brasília, v.22, n.2, p.290-294, 2004.

VIEIRA, E. L. Ação de bioestimulante na germinação de sementes, vigor de plântulas, crescimento radicular e produtividade de soja (Glycine max (L.) Merrill), feijoeiro (Phaseolus vulgaris L.) e arroz (Oryza sativa L.). Piracicaba: Escola Superior de Agricultura Luiz de Queiroz, Universidade de São Paulo, 2001. 122p. Tese (Doutorado).

VIEIRA, E. L., CASTRO, P. R. C. Ação de bioestimulante na germinação de sementes, vigor de plântulas, crescimento radicular e produtividade de soja. Revista Brasileira de Sementes, Brasília, v.23, n.2, p.222-228, 2001.

VIEIRA, E. L., SANTOS, C. M. G. Efeito de bioestimulante no crescimento e desenvolvimento inicial de plantas de algodoeiro. Magistra, Cruz das Almas, v.17, n.3, p.124-130, 2005.

YAMADA, D. Fitorreguladores. In: CASTRO, C. E. F. (ed.). Manual de floricultura. I Simpósio Brasileiro de Floricultura e Plantas Ornamentais. Maringá: SBF, 1992. 279p.

ZIETEMANN, C., ROBERTO, S. R. Efeito de diferentes substratos e épocas de coleta no enraizamento de estacas herbáceas de goiabeira cvs. Paluma e Século XXI. Revista Brasileira de Fruticultura, Jaboticabal, v.29, n.1, p.31-36, 2007. 
Tabela 1. Características químicas dos substratos utilizados no experimento. Campina Grande, 2008. Table 1. Chemical characteristics of the substrates used in the experiment. Campina Grande, 2008.

\begin{tabular}{|c|c|c|c|c|}
\hline \multicolumn{5}{|c|}{ SUBSTRATOS } \\
\hline Caracteristicas Quimicas & $\mathrm{S}_{1}$ & $\mathrm{~S}_{2}$ & $\mathrm{~S}_{3}$ & $\mathrm{~S}_{4}$ \\
\hline \multicolumn{5}{|c|}{ Cátions $\left(\mathrm{cmol}_{\mathrm{c}} \cdot \mathrm{kg}^{-1}\right)$} \\
\hline Cálcio & 33,25 & 6,74 & 2,66 & 2,42 \\
\hline Magnésio & 18,45 & 5,05 & 4,57 & 6,27 \\
\hline Sódio & 0,4 & 0,12 & 0,24 & 0,4 \\
\hline Potássio & 4,4 & 0,86 & 2,11 & 2,82 \\
\hline Hidrogênio & 25,87 & 5,353 & 0 & 0 \\
\hline Aluminio & 0,51 & 0,32 & 0 & 0 \\
\hline
\end{tabular}

\begin{tabular}{|c|c|c|c|c|}
\hline \multicolumn{5}{|c|}{ SUBSTRATES } \\
\hline Chemical characteristics & $\mathrm{S}_{1}$ & $\mathrm{~S}_{2}$ & $\mathrm{~S}_{3}$ & $\mathrm{~S}_{4}$ \\
\hline \multicolumn{5}{|c|}{ Cations ( $\mathrm{cmol}_{\mathrm{c}} \cdot \mathrm{kg}^{-1}$ ) } \\
\hline Calcium & 33,25 & 6,74 & 2,66 & 2,42 \\
\hline Magnesium & 18,45 & 5,05 & 4,57 & 6,27 \\
\hline Sodium & 0,4 & 0,12 & 0,24 & 0,4 \\
\hline Potassium & 4,4 & 0,86 & 2,11 & 2,82 \\
\hline Hydrogen & 25,87 & 5,353 & 0 & 0 \\
\hline Aluminium & 0,51 & 0,32 & 0 & 0 \\
\hline
\end{tabular}

\begin{tabular}{lrrrr}
\multicolumn{5}{c}{ Capacidade de Troca Catiônica } \\
\hline Fosforo assimilável (mg/ 100g) & 51,98 & 20,94 & 12,45 & 21,57 \\
pH (1:2,5) & 4,44 & 4,72 & 7,66 & 7,97 \\
\% Carbono Orgânico & 19,99 & 9,65 & 3,45 & 2,18 \\
\% Matéria Orgânica & 34,46 & 16,64 & 5,64 & 3,76 \\
\% Nitrogênio & 3,45 & 1,66 & 0,56 & 0,38 \\
\hline
\end{tabular}

\begin{tabular}{lrrrr}
\hline \multicolumn{5}{c}{ Cation Exchange Capacity } \\
\hline Assimilable Phosphorus (mg/ 100g) & 51,98 & 20,94 & 12,45 & 21,57 \\
$\mathrm{pH}(1: 2,5)$ & 4,44 & 4,72 & 7,66 & 7,97 \\
\% Organic Carbon & 19,99 & 9,65 & 3,45 & 2,18 \\
\% Organic Matter & 34,46 & 16,64 & 5,64 & 3,76 \\
\% Nitrogen & 3,45 & 1,66 & 0,56 & 0,38 \\
\hline \multicolumn{4}{c}{ Extract of Saturation $\left(\mathrm{mmol}_{-} \mathrm{L}^{-1}\right)$} \\
\hline Chloride & 7,15 & 7,05 & 31,45 & 35,9 \\
Carbonate & 0 & 0 & 0 & 0 \\
Bicarbonate & 4,94 & 4,52 & 15,84 & 21,9 \\
Sulfate & $\mathrm{Abs}$. & $\mathrm{Abs}$. & $\mathrm{Abs}$. & $\mathrm{Abs}$. \\
Calcium & 37,73 & 29,68 & 7,03 & 9,73 \\
Magnesium & 49,75 & 37,75 & 23,65 & 26,3 \\
Potassium & 10,91 & 7,22 & 17,6 & 28,16 \\
\hline pH (Extrat of saturation) & 4,4 & 4,49 & 7,03 & 7,23 \\
EC (dS. m ${ }^{-1}$ ) (Extrat of saturation) & 8,6 & 6,58 & 4,65 & 6,4 \\
\% Saturation & 132,3 & 67 & 29 & 42 \\
\hline
\end{tabular}

$\mathrm{S}_{1}-100 \%$ de substrato comercial Topstrato Hortaliças ${ }^{\circledR} ; \mathrm{S}_{2}-50 \%$, em medida de volume, de substrato comercial misturado com $50 \%$ de solo franco argiloso; $\mathrm{S}_{3}$ $55 \%$ de solo franco argiloso misturado com $15 \%$ de areia lavada, $15 \%$ de esterco bovino curtido e $15 \%$ de maravalha seca, em medida de volume; $\mathrm{S}_{4}$ - $25 \%$ de solo franco argiloso misturado com $25 \%$ de areia lavada, $25 \%$ de esterco bovino curtido e $25 \%$ de maravalha seca.

$S_{1}$ - commercial substrate Topstrato Hortaliças ${ }^{\circledR} ; S_{2}-50 \%$ of commercial substrate mixed with $50 \%$ of clayloam soil; $S_{3}-55 \%$ of clayloam soil mixed with $15 \%$ of washed sand, $15 \%$ of cattle manure and $15 \%$ of wood shavings, and $S_{4}-25 \%$ of clayloam soil mixed with $25 \%$ of washed sand, $25 \%$ of cattle manure and $25 \%$ of wood shavings, all on volume basis.

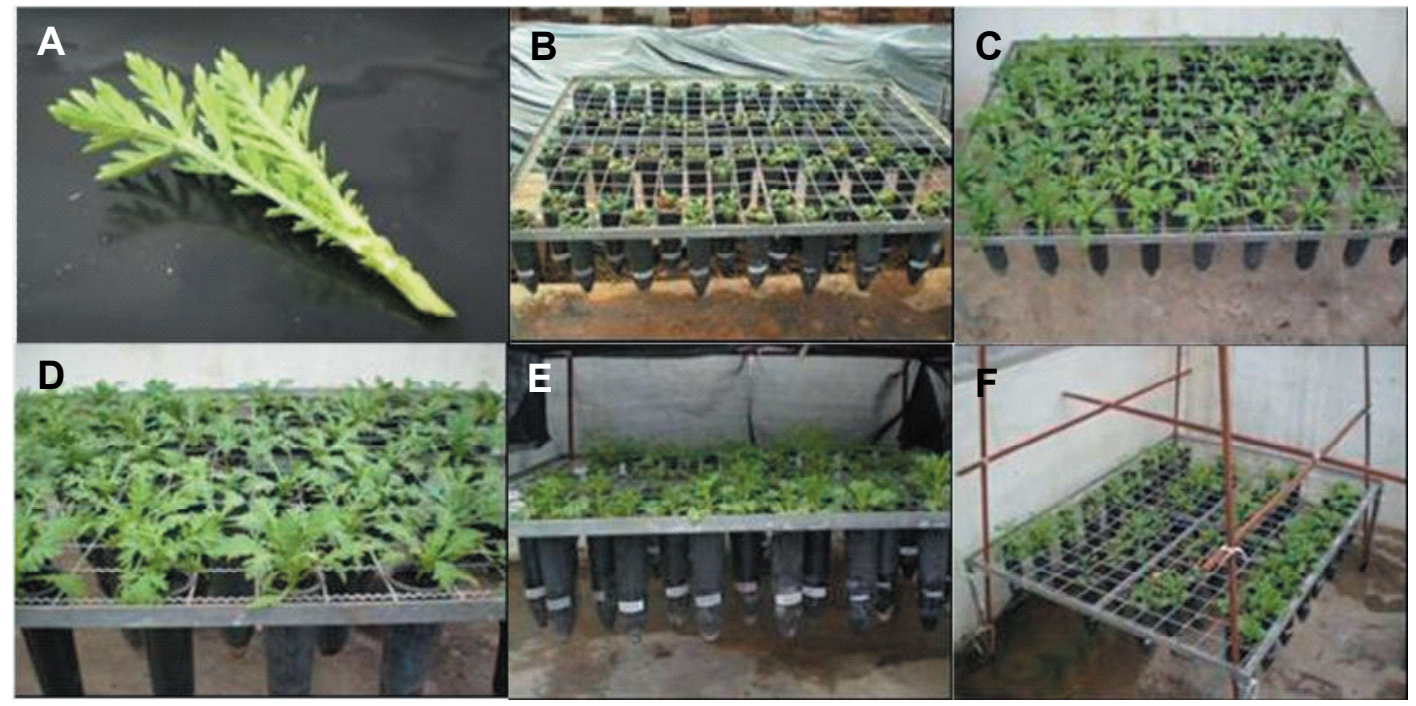

Figura 1: A. Estaca padrão para propagação. B. Estante de tubetes com estacas no primeiro dia após o plantio, ainda murchas. C e D. Visão das mudas se desenvolvendo. E e F. Esquema de cobertura com tela preta e armação, respectivamente.

Figure 1 A. Standard cutting for propagation. B. Poly tube tray with cuttings on first day after planting. C and D. Development of seedlings. E and F. Screened scheme with black covering and wood support, respectively. 
Tabela 2. Características químicas do solo $(0-20 \mathrm{~cm})$ no início do experimento. UFCG, Campina Grande, 2008.

Table 2. Chemical characteristics of the soil (0-20 cm) in the beginning of the experiment. UFCG, Campina Grande, 2008.

\begin{tabular}{|c|c|}
\hline \multicolumn{2}{|l|}{ SOLO } \\
\hline \multicolumn{2}{|l|}{ Caracteristicas Quimicas } \\
\hline \multicolumn{2}{|l|}{ Câtions $\left(\mathrm{cmol}_{\varsigma} \cdot \mathrm{kg}^{-1}\right)$} \\
\hline Câlcio & 1,05 \\
\hline Magnésio & 1,71 \\
\hline Sódio & 0,04 \\
\hline Potássio & 0,34 \\
\hline Hidrogênio & 0,891 \\
\hline Aluminio & 0,15 \\
\hline \multicolumn{2}{|c|}{ Capacidade de Troca Catiônica } \\
\hline Fosforo assimilável (mg/ $100 \mathrm{~g})$ & 1,17 \\
\hline $\mathrm{pH}(1: 2,5)$ & 6,54 \\
\hline \% C. Orgânico & 0,36 \\
\hline \% Matéria Orgânica & 0,62 \\
\hline \% Nitrogênio & 0,06 \\
\hline \multicolumn{2}{|c|}{ Extrato de satuzração $\left(\mathrm{mmol}_{c} \cdot \mathrm{L}^{-1}\right)$} \\
\hline Cloreta & 4,2 \\
\hline Carbonato & 0 \\
\hline Bicarbonato & 2,48 \\
\hline Sulfato & Sus. \\
\hline Câlcio & 0,62 \\
\hline Magnésio & 6,65 \\
\hline Potássio & 0,88 \\
\hline pH (Extrato de saturação) & 5,98 \\
\hline $\mathrm{CE}\left(\mathrm{dS} \cdot \mathrm{m}^{-1}\right)$ (Extrato de saturação) & 0,506 \\
\hline \% Saturação & 23,43 \\
\hline
\end{tabular}

\begin{tabular}{|c|c|}
\hline \multicolumn{2}{|l|}{ SOLO } \\
\hline \multicolumn{2}{|l|}{ Chemical Characteristics } \\
\hline \multicolumn{2}{|l|}{ Cations (cmol. $\mathrm{kg}^{-1}$ ) } \\
\hline Calcium & 1,05 \\
\hline Magnesium & 1,71 \\
\hline Sodium & 0,04 \\
\hline Potassium & 0,34 \\
\hline Hydrogen & 0,891 \\
\hline Aluminium & 0,15 \\
\hline \multicolumn{2}{|l|}{ Cation Exchange Capacity } \\
\hline Assimilable Phosphorus (rng/100g) & 1,17 \\
\hline $\mathrm{pH}(1: 2,5)$ & 6,54 \\
\hline$\%$ Organic Carbon & 0,36 \\
\hline$\%$ Organic Matter & 0,62 \\
\hline$\%$ Nitrogen & 0,06 \\
\hline \multicolumn{2}{|c|}{ Extract of saturation $\left(\mathrm{mmol}_{c} \cdot \mathrm{L}^{-1}\right)$} \\
\hline Chloride & 4,2 \\
\hline Carbonate & 0 \\
\hline Bicarbonate & 2,48 \\
\hline Sulfate & Sus. \\
\hline Calcium & 0,62 \\
\hline Magnesium & 6,65 \\
\hline Potassium & 0,88 \\
\hline $\mathrm{pH}$ (Extrat of saturation) & 5,98 \\
\hline $\mathrm{EC}\left(\mathrm{dS} . \mathrm{m}^{-1}\right)$ (Extrat of saturation) & 0,506 \\
\hline$\%$ Saturation & 23,43 \\
\hline
\end{tabular}

Tabela 3. Resumo das análises de variância para altura de plantas (AP), número de folhas (NF) e comprimento da raiz (CR) aos 30 dias após propagação.

Table 3. Summary of analysis of variance for plant height (AP), number of leaves (NF) and root length (CR) on 30 days after propagation

\begin{tabular}{lcccc}
\hline & \multicolumn{4}{c}{ Quadrados médio } \\
\cline { 3 - 5 } Fonte de variação GL & ${ }^{(1)} \mathrm{AP}$ & ${ }^{(1)} \mathrm{CR}$ & ${ }^{(1)} \mathrm{NF}$ \\
\hline Substrato (S) & 3 & $1,116^{* *}$ & $1,473^{* *}$ & $0,309^{\mathrm{ns}}$ \\
Estimulante (E) & 1 & $0,574^{\mathrm{ns}}$ & $0,607^{\mathrm{ns}}$ & $0,794^{* *}$ \\
Inter S x E & 3 & $0,045^{\mathrm{ns}}$ & $0,128^{\mathrm{ns}}$ & $0,019^{\mathrm{ns}}$ \\
Bloco & 3 & $0,075^{\mathrm{ns}}$ & $0,367^{\mathrm{ns}}$ & $0,038^{\mathrm{ns}}$ \\
Resíduo & 21 & 0,149 & 0,219 & 0,113 \\
\hline CV (\%) & & 19,14 & 10,85 & 8,81 \\
\hline
\end{tabular}

\begin{tabular}{lcccc}
\hline & \multicolumn{4}{c}{ Mean Square } \\
\cline { 3 - 5 } Font of variation & DF & ${ }^{(1)} \mathrm{AP}$ & ${ }^{(1)} \mathrm{CR}$ & ${ }^{(1)} \mathrm{NF}$ \\
\hline Substrate (S) & 3 & $1,116^{* *}$ & $1,473^{* *}$ & $0,309^{\mathrm{ns}}$ \\
Stimulate (E) & 1 & $0,574^{\mathrm{ns}}$ & $0,607^{\mathrm{ns}}$ & $0,794^{* *}$ \\
Inter S x S & 3 & $0,045^{\mathrm{ns}}$ & $0,128^{\mathrm{ns}}$ & $0,019^{\mathrm{ns}}$ \\
Block & 3 & $0,075^{\mathrm{ns}}$ & $0,367^{\mathrm{ns}}$ & $0,038^{\mathrm{ns}}$ \\
Residual & 21 & 0,149 & 0,219 & 0,113 \\
\hline VC (\%) & & 19,14 & 10,85 & 8,81 \\
\hline
\end{tabular}

ns, ** não significativo, significativo a 0,01 de probabilidade, pelo teste $\mathrm{F} .{ }^{(1)}$ Análise realizada após transformação de dados em raiz quadrada de $\mathrm{X}+1$.

ns, ** not significant, significant at 0.01 of probability, by the $F$ test. ${ }^{(1)}$ Transformed data in $X+1$ square root. 


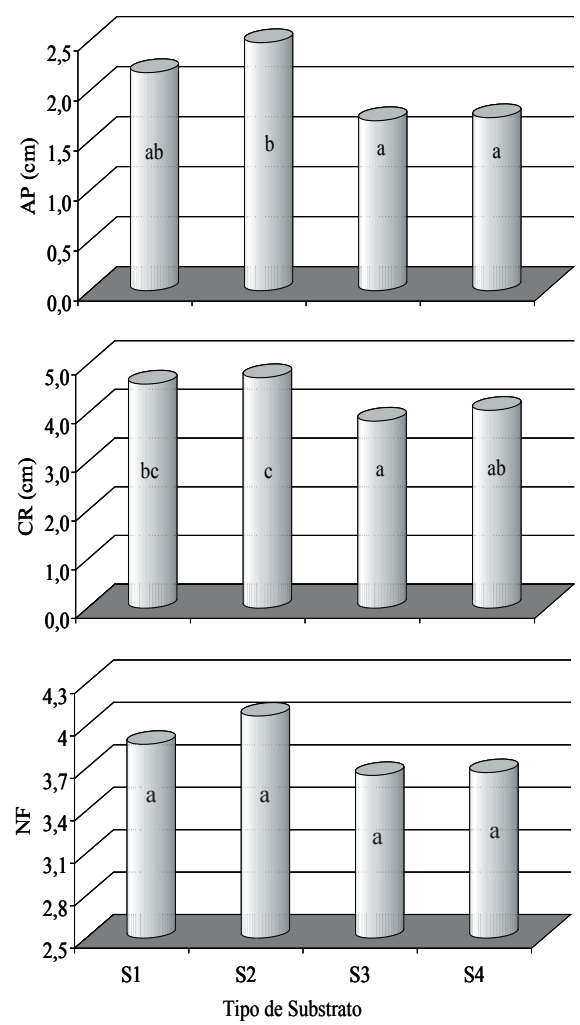

Figura 2: Altura de planta (AP), número de folhas (NF) e comprimento da raiz (CR) do crisântemo em função do substrato ( $\mathrm{S}_{1}-100 \%$ de substrato comercial Topstrato Hortaliças ${ }^{\circledR} ; \mathrm{S}_{2}-50 \%$, em medida de volume, de substrato comercial misturado com $50 \%$ de solo franco argiloso; $\mathrm{S}_{3}-55 \%$ de solo franco argiloso misturado com $15 \%$ de areia lavada, $15 \%$ de esterco bovino curtido e $15 \%$ de maravalha seca, em medida de volume; $\mathrm{S}_{4}-25 \%$ de solo franco argiloso misturado com $25 \%$ de areia lavada, $25 \%$ de esterco bovino curtido e $25 \%$ de maravalha seca). Médias seguidas de mesma letra não diferem estatisticamente entre si pelo teste de Tukey a $1 \%$ de probabilidade.

Figure 2. Plant height (AP), number of leaves (NF) and root length (CR) of chrysanthemum as a function of substrate(S - commercial substrate Topstrato Hortaliças $^{\circledR} ; S_{2}-50 \%$ of commercial substrate mixed with $50 \%$ of clay loam soil; $S_{3}$ - $55 \%$ of clay loam soil mixed with $15 \%$ of washed sand, $15 \%$ of cattle manure and $15 \%$ of wood shavings, and $S_{4}-25 \%$ of clay loam soil mixed with $25 \%$ of washed sand, $25 \%$ of cattle manure and $25 \%$ of wood shavings, all on volume basis). Means followed by the same letter do not differ statistically between themselves. 


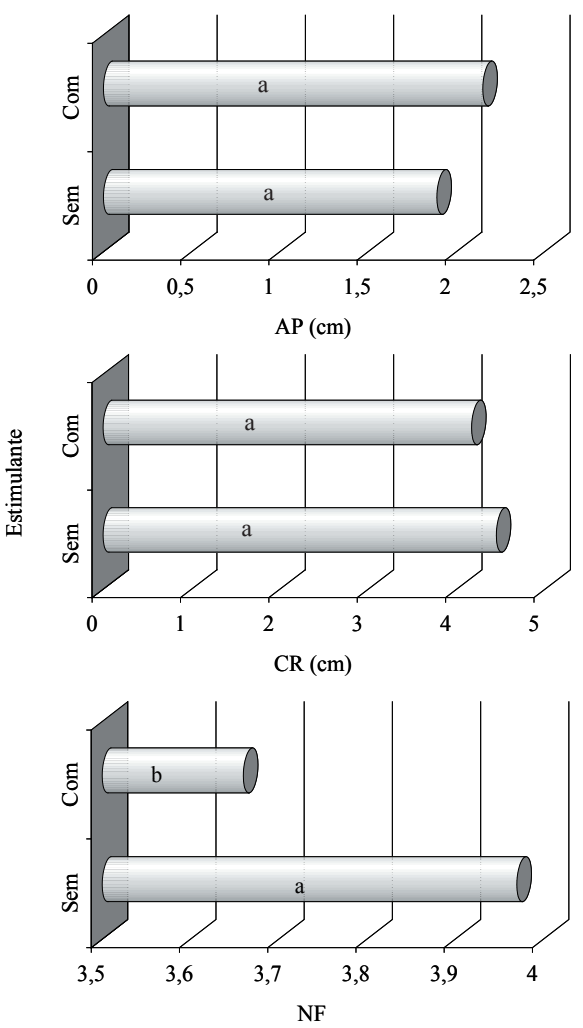

Figura 3: Altura de planta (AP), número de folhas (NF) e comprimento da raiz (CR) do crisântemo em função da utilização de estimulante. Médias seguidas de mesma letra não diferem estatisticamente entre si pelo teste de Tukey a $1 \%$ de probabilidade.

Figure 3. Plant height (AP), number of leaves (NF) and root length (CR) of chrysanthemum as a function of use of growth promoter. Means followed by the same letter do not differ statistically between themselves.

Tabela 4. Resumo das análises de variância para massas de matérias fresca e seca da parte aérea (FFPA e FSPA), massas de matérias fresca e seca da raiz (FFR e FSR) e massas de matérias fresca e seca total (FFT e FST), na fase inicial do crisântemo. Table 4. Summary of analysis of variance of fresh and dry weight of aerial parts (FFPA e FSPA), fresh and dry weight of root part (FFR e FSR) and fresh and dry total weight (FFT e FST), in the initial phase of chrysanthemum.

\begin{tabular}{|c|c|c|c|c|c|c|c|}
\hline \multirow[b]{2}{*}{ Fonte de variação } & \multirow{2}{*}{ GL } & \multicolumn{6}{|c|}{ Quadrados médio (1) } \\
\hline & & FFPA & FFR & FFT & FSPA & FSR & FST \\
\hline Substrato (S) & 3 & $0,202^{\mathrm{ns}}$ & $0,041^{\mathrm{ns}}$ & $0,291^{\mathrm{ns}}$ & $0,001^{\mathrm{ns}}$ & $0,0001^{\mathrm{ns}}$ & $0,0013^{\mathrm{ns}}$ \\
\hline Estimulante (E) & 1 & $1,178 * *$ & $0,140 * *$ & $1,558 * *$ & $0,012 * *$ & $0,0009 * *$ & $0,0185 * *$ \\
\hline Inter $\mathrm{S} \times \mathrm{E}$ & 3 & $0,030^{\mathrm{ns}}$ & $0,009^{\mathrm{ns}}$ & $0,049^{n s}$ & $0,001^{n s}$ & $0,0001^{\mathrm{ns}}$ & $0,0007^{\mathrm{ns}}$ \\
\hline Bloco & 3 & $0,080^{\mathrm{ns}}$ & $0,006^{\mathrm{ns}}$ & $0,014^{\mathrm{ns}}$ & $0,001^{\mathrm{ns}}$ & $0,0001^{\mathrm{ns}}$ & $0,0002^{\mathrm{ns}}$ \\
\hline Resíduo & 21 & 0,067 & 0,019 & $0,103^{\mathrm{ns}}$ & 0,001 & 0,0002 & 0,0014 \\
\hline $\mathrm{CV}(\%)$ & & 14,66 & 10,77 & 16,59 & 2,6 & 1,22 & 3,37 \\
\hline
\end{tabular}

\begin{tabular}{|c|c|c|c|c|c|c|c|}
\hline \multirow[b]{2}{*}{ Font of variation } & \multirow{2}{*}{ DF } & \multicolumn{6}{|c|}{ Mean Sauare ${ }^{(1)}$} \\
\hline & & FFPA & FFR & FFT & FSPA & FSR & FST \\
\hline Substrate (S) & 3 & $0,202^{\mathrm{ns}}$ & $0,041^{\mathrm{ns}}$ & $0,291^{\mathrm{ns}}$ & $0,001^{n s}$ & $0,0001^{\mathrm{ns}}$ & $0,0013^{\mathrm{ns}}$ \\
\hline Stimulate (E) & 1 & $1,178 * *$ & $0,140 * *$ & $1,558 * *$ & $0,012 * *$ & $0,0009 * *$ & $0,0185 * *$ \\
\hline Inter $\mathrm{S} \times \mathrm{S}$ & 3 & $0,030^{\mathrm{ns}}$ & $0,009^{\mathrm{ns}}$ & $0,049^{n s}$ & $0,001^{n s}$ & $0,0001^{\mathrm{ns}}$ & $0,0007^{\mathrm{ns}}$ \\
\hline Block & 3 & $0,080^{\mathrm{ns}}$ & $0,006^{\mathrm{ns}}$ & $0,014^{\mathrm{ns}}$ & $0,001^{n s}$ & $0,0001^{\mathrm{ns}}$ & $0,0002^{\mathrm{ns}}$ \\
\hline Residual & 21 & 0,067 & 0,019 & $0,103^{\mathrm{ns}}$ & 0,001 & 0,0002 & 0,0014 \\
\hline $\mathrm{VC}(\%)$ & & 14,66 & 10,77 & 16,59 & 2,6 & 1,22 & 3,37 \\
\hline
\end{tabular}

(1) ns, ** não significativo, significativo de 0,01 de probabilidade, pelo teste $\mathrm{F}$. Análise realizada após transformação de dados em raiz quadrada de $\mathrm{X}+1$.

ns, **not significant, significant on 0.01 of probability, by the $F$ test. Transformed data in $X+1$ square root. 

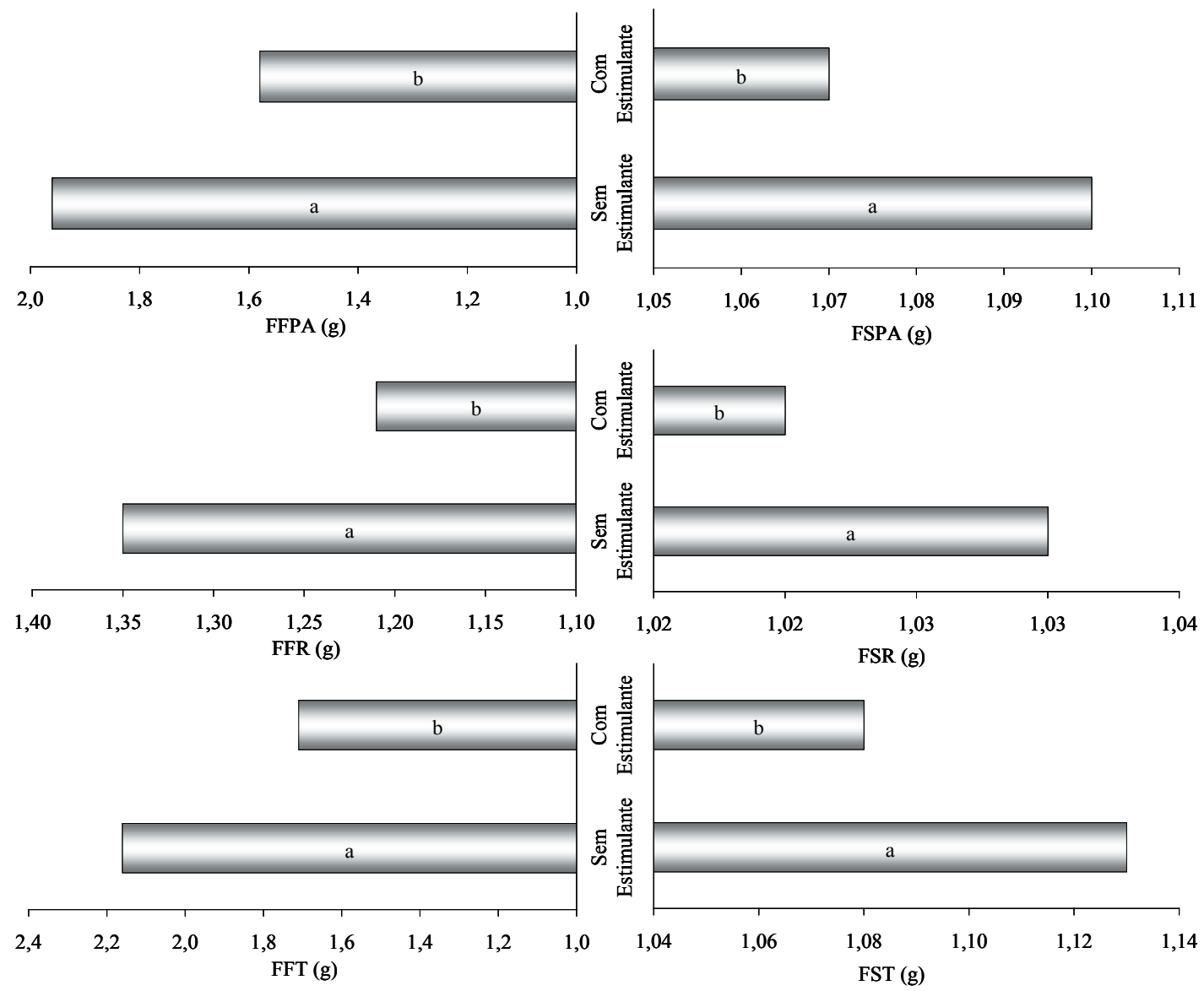

Figura 4: Massas de matérias fresca e seca da parte aérea (FFPA e FSPA), da raiz (FFR e FSR) e total (FFT e FST) do crisântemo em função da aplicação de estimulante. Médias seguidas de mesma letra não diferem estatisticamente entre si pelo teste de Tukey $1 \%$ de probabilidade.

Figure 4. Plant fresh and dry weight of the aerial part (FFPA and FSPA), root (FFR and FSR) and total (FFT and FST) of chrysanthemum as a function of use of growth promoter. Means followed by the same letter do not differ statistically between themselves. 